\title{
The effect of stellar limb darkening values on the accuracy of the planet radii derived from photometric transit observations
}

\author{
Sz. Csizmadia ${ }^{1}$, Th. Pasternacki ${ }^{1}$, C. Dreyer ${ }^{1,2}$, J. Cabrera ${ }^{1}$, A. Erikson ${ }^{1}$, and H. Rauer ${ }^{1,2}$ \\ ${ }^{1}$ Institute of Planetary Research, German Aerospace Center, Rutherfordstrasse 2, 12489 Berlin, Germany \\ e-mail: szilard.csizmadia@dlr.de \\ 2 Zentrum für Astronomie und Astrophysik, TU Berlin, Hardenbergstrasse 36, 10623 Berlin, Germany
}

Received 25 June 2012 / Accepted 7 September 2012

\begin{abstract}
Context. The radius of an exoplanet is one of its most important parameters. Studies of planetary interiors and their evolution require $1 \%$ precision in the radius determination. Transiting exoplanets offer a unique oppurtunity to measure the radius of exoplanets in stellar units. These radius measurements and their precision are strongly affected by our knowledge of limb darkening.

Aims. We study how the precision of the exoplanet radius determination is affected by our present knowledge of limb darkening in two cases: when we fix the limb darkening coefficients and when we adjust them. We also investigate the effects of spots in one-colour photometry.

Methods. We study the effect of limb darkening on the planetary radius determination both via analytical expressions and by numerical experiments. We also compare some of the existing limb darkening tables. When stellar spots affect the fit, we replace the limb darkening coefficients, calculated for the unspotted cases, with effective limb darkening coefficients to describe the effect of the spots. Results. There are two important cases. (1) When one fixes the limb darkening values according to some theoretical predictions, the inconsistencies of the tables do not allow us to reach accuracy in the planetary radius of better than 1-10\% (depending on the impact parameter) if the host star's surface effective temperature is higher than $5000 \mathrm{~K}$. Below $5000 \mathrm{~K}$ the radius ratio determination may contain even $20 \%$ error. (2) When one allows adjustment of the limb darkening coefficients, the $a / R_{\mathrm{S}}$ ratio, the planet-to-stellar radius ratio, and the impact parameter can be determined with sufficient accuracy $(<1 \%)$, if the signal-to-noise ratio is high enough. However, the presence of stellar spots and faculae can destroy the agreement between the limb darkening tables and the fitted limb darkening coefficients, but this does not affect the precision of the planet radius determination. We also find that it is necessary to fit the contamination factor, too.

Conclusions. We conclude that the present inconsistencies of theoretical stellar limb darkening tables suggests one should not fix the limb darkening coefficients. When one allows them to be adjusted, then the planet radius, impact parameter, and the $a / R_{\mathrm{S}}$ can be obtained with the required precision.
\end{abstract}

Key words. techniques: photometric - planetary systems

\section{Introduction}

The depth of an exoplanetary transit is primarily determined by the ratio of the radius of the transiting exoplanet to the host star. Limb darkening is a second-order, but key effect for determining the exact radius of the planet, because limb darkening not only modifies the shape of the transit light curve, but it also significantly affects the true transit depth. There are other thirdorder effects (e.g. night-side radiation, exorings, gravity darkening, etc.), but they are not studied here.

Transit parameters are determined by fitting a model to the observed light curve data points. Typically the number of free parameters is around seven (e.g. epoch, period, duration of the transit, the impact parameter, radius ratio of the planet and the host star, and two limb darkening coefficients). If we knew a priori the exact values of the limb darkening coefficients, then the number of free parameters would be significantly reduced by fixing the limb darkening coefficients. This would mean not only that the dimensions of this optimization problem are reduced, but also that the occasional degeneracy of the fitting procedure reported by several authors would disappear (e.g. Brown et al. 2001; Deleuil et al. 2008; Csizmadia et al. 2011).
Values of the limb darkening coefficients have been published by several authors for many photometric passbands as a function of the stellar effective temperature, metallicity, $\log g$, and turbulent velocity in the stellar atmosphere. Such tables can be found in van Hamme (1993), Diaz-Cordoves et al. (1995), Claret et al. (1995), Claret (2000, 2004), Claret \& Hauschildt (2003), Barban (2003), Sing (2010), Howarth (2011), and Claret $\&$ Bloemen (2011). For earlier tables see the summary of van Hamme (1993).

Some investigators of transit light curves have found good agreement between theoretical predictions and fitted limb darkening coefficients (e.g. in CoRoT-8, Bordé et al 2010, or in CoRoT-11 Gandolfi et al. 2010); however, sometimes larger differences were found (e.g. at $2 \sigma$ level in CoRoT-13, Cabrera et al. 2010) or the fit did not constrain the values of the limb darkening coefficients at all (e.g. in CoRoT-12 Gillon 2010). Complete disagreement can often be found between the theoretically predicted and the observed limb darkening coefficients, even where the quality of the light curve is extremely good (e.g. the analysis of the HST light curve of HD 209458 by Claret 2009, reanalysis of Kepler-5b by Kipping \& Bakos 2011); or else, the ground-based photometry of WASP-13 by (Barros et al. 2012), 
who also point out that these discrepancies are not caused by the photometric signal-to-noise ratio $(\mathrm{S} / \mathrm{N})$, and the quality of the light curves are sufficient to say that aforementioned discrepancies are real. Claret (2009) concludes that uncertainties in the stellar parameters (effective temperature, surface gravity $(\log g)$, metallicity) are also not responsible for the discrepancies.

Neilson \& Lester (2011) point out that the used plan-parallel theory might be replaced by spherical symmetry for calculating limb darkening coefficients, which may provide better predictions for limb darkening. The 3D stellar atmosphere models also offer an oppurtunity to improve the predictions. However, these very new models have not been tested against full and multicolour light curves yet (Hayek et al. 2012).

Since limb darkening changes true transit depths (see appendix), the question arises: do these insonsistencies affect the accuracy of the transit parameter determination? As we show, the answer is yes. In addition, to distinguish between different planetary models and to study them in detail we require $\pm 1 \%$ precision in planetary radii below five Earth masses (Wagner et al. 2011; F. Sohl, priv. comm.), a level that cannot be reached in all temperature regions when fixing the limb darkening coefficients for the transit fit.

The aim of this paper is not only to take a step toward better understanding what can cause inconsistent observed limb darkening coefficients. We also want to make certain that such inconsistencies between observed and predicted limb darkening coefficients do not affect the determination of transit parameters.

In Sect. 2 we study the differences between recent limb darkening tables. In Sect. 3 we investigate the effect of the poor knowledge of the limb darkening on the planet-to-stellar radius ratio. In Sect. 4 we check the accuracy of the adjusted limb darkening coefficients and study this on synthetic data. We call attention to the impact of stellar spots on the transit fits and their importance in the observable limb darkening coefficients in Sect. 5. We check the determinability of the limb darkening coefficients in Sect. 6. Our conclusions can be found in Sect. 7.

\section{Comparison of theoretical predictions for limb darkening coefficients}

In Fig. 1 the limb darkening coefficients predictions of Sing (2010) and Claret \& Bloeman (2011) are plotted for three different stellar metallicities: $[M / H]=-0.5,0.0,1.0$. It is obvious that the values given by these different authors deviate from each other by a value of $0.01-0.3$, depending on which temperature region we consider. The usual range of the limb darkening coefficients is between approximately -0.1 and 0.8 , so these differences are considered as large.

We concentrate on the 3500-8000 K temperature range, because most of the transiting exoplanets have been detected around main sequence stars with such temperatures. Interestingly, we have better agreement between models in the high-metallicity range $([M / H]=1.0)$. Here the coefficients agree with each other, within 0.02 at the low-temperature end, but over $7000 \mathrm{~K}$, they differ from each other by 0.05 . Fortunately, this temperature region is less interesting for transit hunters. At intermediate temperatures the predictions for limb darkening coefficients do not differ by more than 0.01 to 0.02 .

At lower metallicities the situation is completely different. Below $\sim 4500 \mathrm{~K}$, the two lower metallicities are in complete disagreement, and the relative differences between the predictions can be as high as 0.3 . Between $\sim 5000 \mathrm{~K}$ and $\sim 8000 \mathrm{~K}$ the situation is still problematic, and in the case of $[M / H]=0.0$ the two tables generally differ by 0.2 . Similar conclusions have also been reached by Claret \& Bloemen (2011).

Some modellers fix the limb darkening coefficients according to these or other tables when they fit a transit curve. As is clear from the aforementioned discrepancies, in some temperature regions this is not a good strategy because the values of the limb darkening coefficients are not consistent and it is not clear which are the correct ones.

When investigators adjust the limb darkening coefficients, then it is customary to compare the results to some limb darkening coefficient table. Unfortunately, most authors compare their results to only one table, and this may not be appropriate. It is necessary to compare the results to all available tables because of the disagreements between tables.

Such a comparison was done by Southworth et al. (2007), among others. After modelling the light curve of an eclipsing binary star, they compared the resulting limb darkening coefficients to the tables of Diaz-Cordoves \& Claret (1995), Claret (2000), and Claret \& Hauschildt (2003). The predicted values of these three tables typically differ from each other by $\sim 40-60 \%$ (see Table 5 of Southworth 2007). In their paper the relative error of the resulting limb darkening coefficients was $\sim 35 \%$, and the limb darkening values observed were consistent with more than one theory. Consequently, they could not decide which theory describes the reality, so the uncertainty limits are inconclusive for distinguishing the theories.

Claret (2009) concluds that no presently available limb darkening calculation is able to reproduce the surface brightness distribution of HD 209458. Claret (2008) also points out that the observations do not support any of the limb darkening tables.

The differences between the different limb darkening tables are mainly due to the adopted numerical methods and not to the atmosphere models themselves. In addition, to have a clue to stellar atmosphere studies, it will be necessary to compare the differences between the observations and theory. This gives another argument for why it is worthy and necessary to fit the limb darkening coefficients.

\section{The effect of poor knowledge of fixed limb darkening coefficients on the radius determination}

Here we investigate the hidden inaccuracy caused by fixing the limb darkening coefficients during the transit-fitting procedure. Our goal is to calculate the error in $k$ caused by the theoretical uncertainties of the limb darkening coefficients. We assume the time being that the normalized transit depth $\Delta F / F$ is perfectly known since we are only interested in the error caused by the inconsistent limb darkening tables.

Let $\Delta u_{1}$ and $\Delta u_{2}$ be the uncertainties of the theoretically calculated linear and the quadratic limb darkening coefficients, respectively. These uncertainties have four sources: (i) the theories have some uncertainties. These kinds of uncertainties are not well known, but as a first approximation, we can assume that their order of magnitude is equal to the differences between different theories as mentioned in Sect. 2; (ii) the input stellar parameters ( $T_{\mathrm{eff}}, \log g$, metallicity) have uncertainties that will be reflected in the prediction of limb darkening coefficients. The uncertainties of stellar parameters usually cause small uncertainties in the prediction of the limb darkening coefficients, and for instance, Deleuil et al. (2012) found that the uncertainty of the predicted limb darkening coefficients was \pm 0.0168 when 


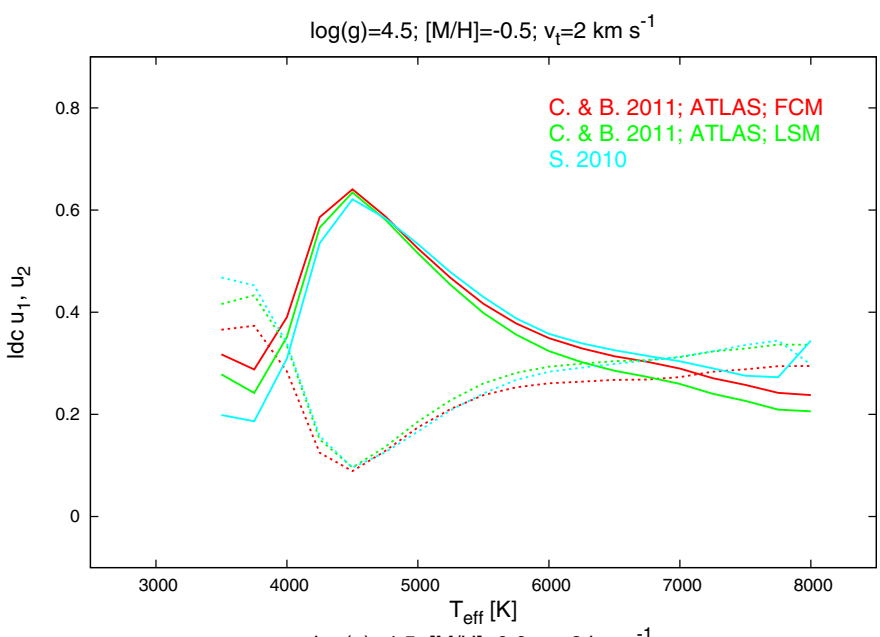

$\log (g)=4.5 ;[M / H]=0.0 ; v_{t}=2 \mathrm{~km} \mathrm{~s}^{-1}$

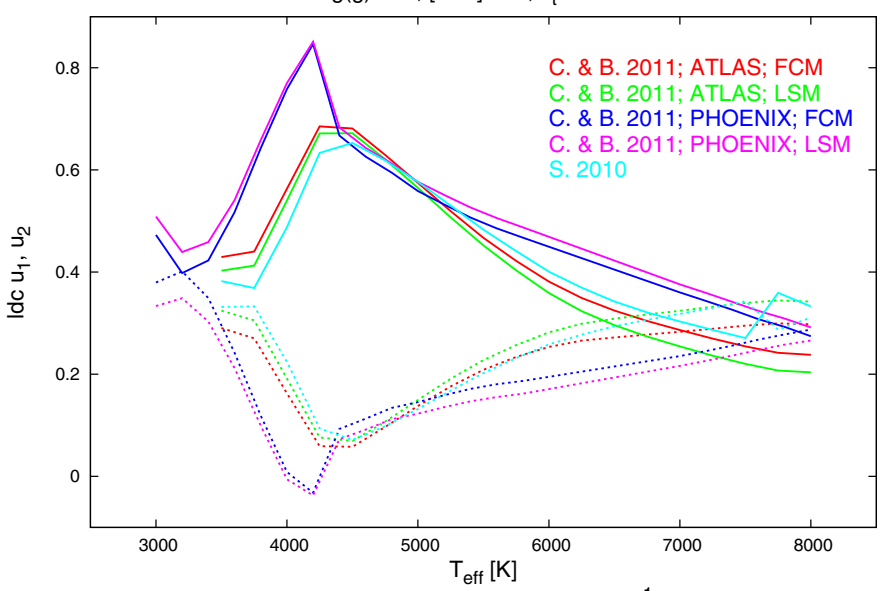

$\log (g)=4.5 ;[M / H]=1.0 ; v_{t}=2 \mathrm{~km} \mathrm{~s}^{-1}$

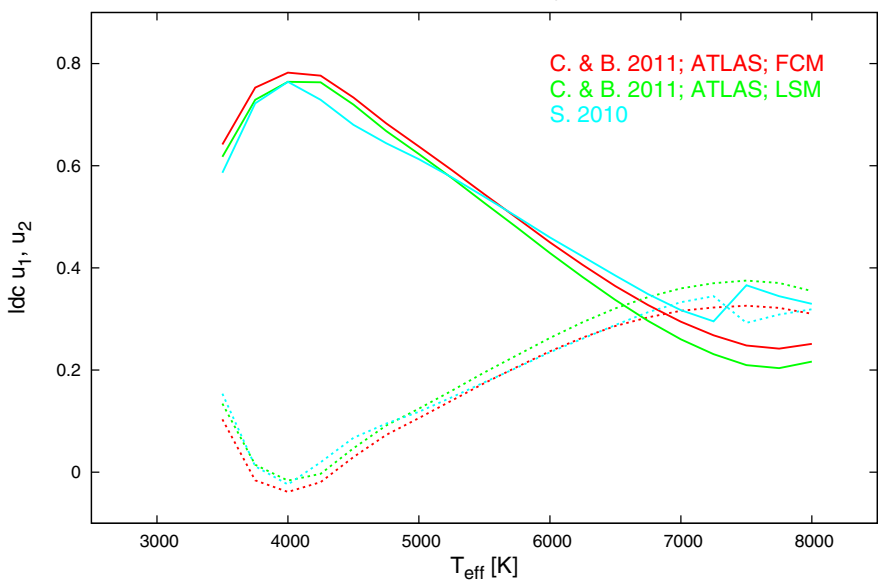

Fig. 1. A comparison of 5 (3) different limb darkening models. We plotted only the coefficients of the quadratic limb darkening-law. From top to bottom one can see the models for different metallicities $([M / H]=$ $-0.5,0.0,1.0)$. For the sake of correct comparison, we used the same $\log$ g and turbulent velocity $v_{t}$ values for all the curves. Solid lines represent the $u_{1}$ coefficients, dotted lines the $u_{2}$ coefficients. The model of Sing (2010) is denoted by cyan lines. Red lines show the models of Claret \& Bloeman (2011), which are based on the ATLAS synthetic spectra and their "flux conservation method" (FCM). Green lines were obtained by them using the same spectra, but applying their "least square method" (LSM) for calculating the limb darkening coefficients. For solar metallicity $([\mathrm{M} / \mathrm{H}]=0.0)$, they also calculated the limb darkening coefficients with these two methods, but from the PHOENIX spectra, too (violet and magenta lines). The figure only hows the CoRoT white light passband coefficients; the discrepancies are similar for Kepler's response function and for other photometric systems. they propagated the stellar parameter uncertainties to the limb darkening prediction. In most of the cases the stellar paramater uncertainties cause less than \pm 0.03 uncertainty in the prediction of the limb darkening coefficients; (iii) although the spectroscopic effective temperature determination is not significantly affected by stellar spots in the case of a solar-like activity, the stellar surface effective temperature determination can be systematically affected when the spot coverage is about $10-20 \%$ (Hatzes, priv. comm.; Ribas et al. 2008), and this can lead to systematic errors in the limb darkening predictions and to underestimation of the uncertainties of such a prediction. Thus, the uncertainties mentioned in point (ii) might be underestimated. It is quite difficult to characterize these kinds of error sources, but fortunately they have an impact only for very active stars, which are usually avoided by planet hunters; (A remarkable exception is CoRoT-2.) (iv) the inhomogeneties of the stellar surface temperature distribution (e.g. spots, faculae, gravity darkeningeffects, etc.) are not included in the prediction of limb darkening coefficients.

Now we consider the uncertainty in the planet-to-stellar radius ratio caused by the uncertainty of the limb darkening coefficients. After straightforward calculation we have (cf. Eq. (B1)):

$\frac{\Delta k}{k}=\frac{1}{2}\left(\frac{\Delta u_{1} \delta+\Delta u_{2} \delta^{2}}{1-u_{1} \delta-u_{2} \delta^{2}}+\frac{2 \Delta u_{1}+\Delta u_{2}}{6-2 u_{1}-u_{2}}\right)$

where we abbreviated $\delta=1-\mu$, and $\Delta x$ means the uncertainty of quantity $x$. The variation in the relative error in the radius ratio of the star and the planet from Eq. (1) is shown in Fig. 2.

First we consider the temperature region $5000 \mathrm{~K}<T<$ $7500 \mathrm{~K}$. Here the different limb darkening theories agree more closely. Substituting the uncertainties of the theory discussed in the previous section, we can easily conclude that the uncertainties in the relative radius ratio of the planet and the star varies between $1 \%$ and $10 \%$, but it can be around $8 \%$ at the high temperature. Below $5000 \mathrm{~K}$, where the curves of different theories are very divergent, we have uncertainties of up to $20 \%$ in the radius ratio. These uncertainties only come from the inconsistencies of the theoretical limb darkening calculations. The aforementioned two additional factors increase these uncertainties further, but one needs a case-to-case study to characterize them.

These uncertainties are unacceptable if radii and therefore internal structure of the planets are being studied, because to distinguish between different planet models and to study them in detail we require $\pm 1 \%$ precision in planetary radii below five Earth-masses. The consistency of the limb darkening-tables should be improved in all temperature regions and should be checked via observations, to obtain more concordant limb darkening tables in the near future.

Since we do not have reliable limb darkening theories it seems questionable to fix the limb darkening coefficients for transit light curve analysis. For instance, CoRoT and Kepler are able to determine the transit depth with a precision of $10^{-4}$ in general or sometimes even better, which can be translated to $0.5 \%$ relative error for the radius ratio $k$ - in the absence of limb darkening. Since the error due to the poor knowledge of the limb darkening may be as big as $20 \%$ at low host-star surface temperatures $(T<5000 \mathrm{~K})$, this can cause $\sim 40-50$ times larger error in the radius than the error stemming from the quality of photometry alone! This error source is also two to ten times bigger at the higher host-star's surface temperatures $(T>5000 \mathrm{~K})$ than the error stemming from the photometry. This is the case when one fixes the limb darkening coefficients during the transit light curve fit procedure. 

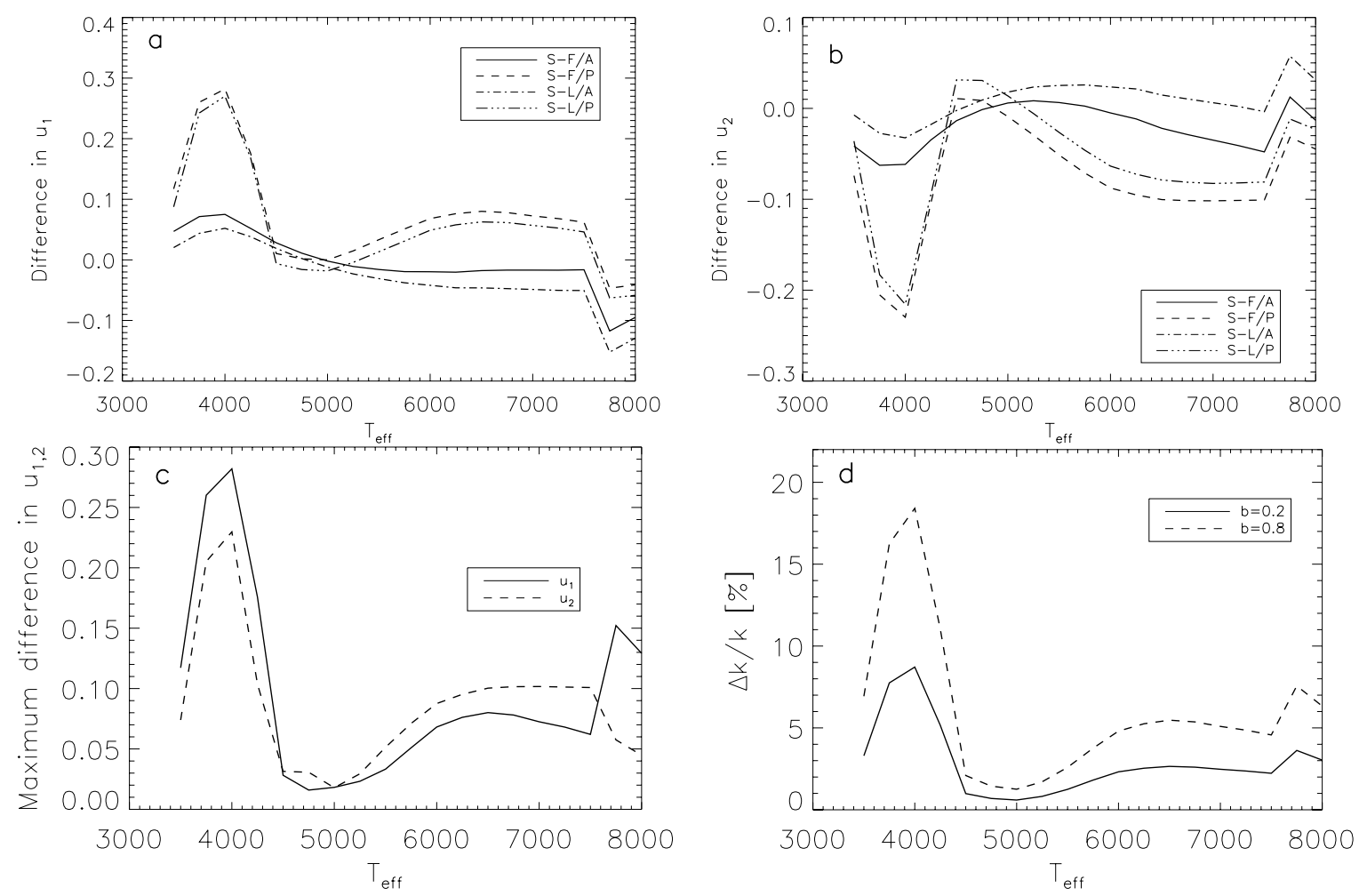

Fig. 2. a) The difference of the theoretically predicted limb darkening coefficient $u_{1}$ as a function of the stellar effective surface temperature between Sing (2010) (S) and different models of Claret \& Bloemen (2011). F means the flux conservation method, L means the least square method of Claret \& Bloemen (2011). A and P denote the ATLAS and the PHOENIX models that they used for creating of their limb darkening tables. b) The same for the $u_{2}$ quadratic coefficients. c) The absolute value of the maximum differences at a given temperature between Sing (2010) and some of the Claret \& Bloemen (2011) models. The curves in this panel are upper limits, because we calculated the differences between the different limb darkening tables in a conservative way; i.e., we searched for the biggest difference between Sing's table and any of the Claret \& Bloemen's table at the given temperature. d) Visualization of the meaning of Eq. (1): relative error of the radius ratio of the star and the planet $k=R_{\text {planet }} / R_{\text {star }}$ vs. effective surface temperature of the stellar surface when the limb darkening coefficients are fixed. The $\Delta u_{1}, \Delta u_{2}$ uncertainties are taken from panel c). The two curves are valid for a rather central $(b=0.2)$ and for a rather grazing $(b=0.8)$ transit. For these plots we used the CoRoT passband limb darkening coefficients of Sing (2010) and Claret \& Bloemen (2011), and we assumed $\log g=4.50$, solar metallicity and a turbulent velocity of $2 \mathrm{~km} \mathrm{~s}^{-1}$. However, the main result does not change significantly for other input parameters or passbands. The uncertainty of the parameters were estimated as the difference between the two limb darkening tables. In certain temperature regions we are far from the required precision in the radius ratio determination.

We add that these numbers come from the optimistic case; i.e., uncertainty in the stellar radius can be neglected. But this is not the case. The aforementioned errors are the errors stemming from the planet-to-stellar radius ratio determination alone. When this radius ratio is transformed to absolute dimensions of the planet via $R_{\text {planet }}=k R_{\text {star }}$, then the error bars should be increased more accordingly, by the uncertainties in the stellar radius. The stellar radius is usually obtained by comparing stellar evolutionary models to the spectroscopically measured quantities, such as $T_{\text {eff }}$ and $\log g$ of the star. It seems that the most careful spectroscopic studies of the host stars are able to yield the stellar radius with a precision not better than $\sim 5 \%$ (e.g. Bruntt et al. 2010; see also the discussion in Torres et al. 2012). However, the final error budget also depends on the uncertainties and systematics of the stellar evolutionary models used. For instance, the radii of stars below $1 M_{\odot}$, predicted by current evolutionary models, are not supported by observations (see e.g. Clausen et al. 2009, and references therein). It is beyond the scope of this paper to characterize these kinds of error sources, we limit our study only to the effect of limb darkening on the planet-to-stellar radius ratio, and therefore we refer to Torres et al. (2010), where the uncertainties of the used stellar models and the derived stellar parameters are discussed. We also mention that future projects, like PLATO, are expected to provide the stellar masses and radii with less than couple of percent relative error via asteroseismological studies. Details of this latter method and its uncertainties are discussed e.g. in Catala (2009).

\section{Adjusting the limb darkening coefficients}

Here we study how precisely our fitting procedure is able to determine the different transit parameters. For this purpose we created 2000 synthetic light curves with the subroutines of Mandel \& Agol (2002). We assumed a circular orbit for all of these curves. The number of the simulated photometric data points inside the transit were selected randomly between 500 and 5000 . Then we added Gaussian-type random noise to these light curves with zero mean and $1 \sigma$ scatter, and we chose randomly $\sigma$ such that our final light curves have $\mathrm{S} / \mathrm{N}$ between 1 and 1000. The S/N is defined as follows: $S / N=k^{2} / \sigma$. We did not study the effect of red noise, systematic errors, etc., since our aim is to find what minimum accuracy is needed for precise parameter determination. The input parameters of these synthetic light curves were randomly chosen between the following limits: $b=0 \ldots 1$, $k=0 \ldots 0.3, a / R_{\mathrm{S}}=5 \ldots 50, u_{+}=0 \ldots 1$ and $u_{-}=-0.1 \ldots 1.0$, respectively. Here, $a$ is the semi-major axis, $R_{\mathrm{S}}$ the stellar 
radius $-a / R_{\mathrm{S}}$ are often called scaled semi-major axes,$- b$ is the impact parameter, and $u_{+}=u_{1}+u_{2}$ and $u_{-}=u_{1}-u_{2}$. The use of these combinations of the limb darkening coefficients was suggested by Brown et al. (2001) and they aim to avoid degeneracies between $u_{1}$ and $u_{2}$. We selected $u_{+}$and $u_{-}$randomly between the aforementioned limits. This means that they are not related in this test to any stellar properties or theoretical considerations, and thus there is no link between them; i.e., they are independent of each other in this test.

In the next step we modelled these artificial light curves with our fitting procedure, which is based on a genetic algorithm (Geem et al. 2011). The free parameters were the impact parameter $b, k, a / R s$ ratio and $u_{+}$. We fixed the value of $u_{-}$at 13 different values at $-0.3,-0.2,-0.1, \ldots, 0.9,1.0$, and then we selected the best solution (according to the $\chi^{2}$-values). Then, this best solution was refined by allowing $u_{-}$to vary in a narrower range $( \pm 0.1)$. After that we plotted the standard devations of the differences between the value obtained from the light curve solution and the original input value, normalized to the input value vs. the $\mathrm{S} / \mathrm{N}$ ratio as it is plotted in Fig. 3:

$s=\operatorname{STD}$ DEV $\left(\frac{\mid \text { modelled value }- \text { input value } \mid}{\text { input value }}\right)$.

This $s$-value has been plotted in the different panels of Fig. 3 against the $\mathrm{S} / \mathrm{N}$ of the simulated light curves. We draw conclusions from this figure as follows. We need at least $S / N=25$ and 6 to determine the limb darkening coefficients and the scaled semi-major axis with $\pm 5 \%$ uncertainty. To determine $k$ with $1 \%$ accuracy, we need at least $S / N=\sim 50$. To determine $b$ with $10 \%$ relative accuracy, we need $S / N=\sim 100$ at least. We also found that when $b>0.85$ the solution becomes unstable and far from the input values, so we removed them from this analysis.

\section{Effect of stellar spots on the limb darkening}

The result of a fit with adjusted limb darkening coefficients can lead to unreasonable limb darkening values, e.g. Southworth (2011) accepted a worse solution for CoRoT-13b in the sense of the quality of the fit (measured by the $\chi^{2}$-values), because when he adjusted both limb darkening coefficients, he obtained "unphysical" values, i.e. far from the theoretically predicted range, sometimes causing "limb brightening" instead of limb darkening or negative fluxes at the edge. What can we say about such cases?

We would like to point out that we employed an indirect assumption in the previous sections: the effective temperature of the stellar surface is assumed to be the same at each point of the surface and therefore the stellar surface brightness distribution is only modified by limb darkening. The same assumption is used when the theoretical limb darkening coefficients are calculated, and almost every transit light curve investigator uses the same assumption. However, there are several factors that modify the local effective temperature on the stellar surface. Such effects are e.g. stellar rotation (i.e. gravity darkening, see e.g. von Zeipel 1924; Twigg \& Raffert 1980; Barnes 2009; Espinoza \& Rieutord 2011; Claret 2012), reflection effect (although we can neglect this for the star in a star-planet system), and stellar activity phenomena: spots, plage-area, faculae and flares. Some of these are transient phenomena, e.g. the flares, but others have similar or much longer timescales than the length of the transits (e.g. the spots and faculae). They can be regarded as an additional, but constant surface brightness distortion during a transit. Constant here means that we assume that the number, temperature, size, astrographic longitude, and latitude of the distortion(s)
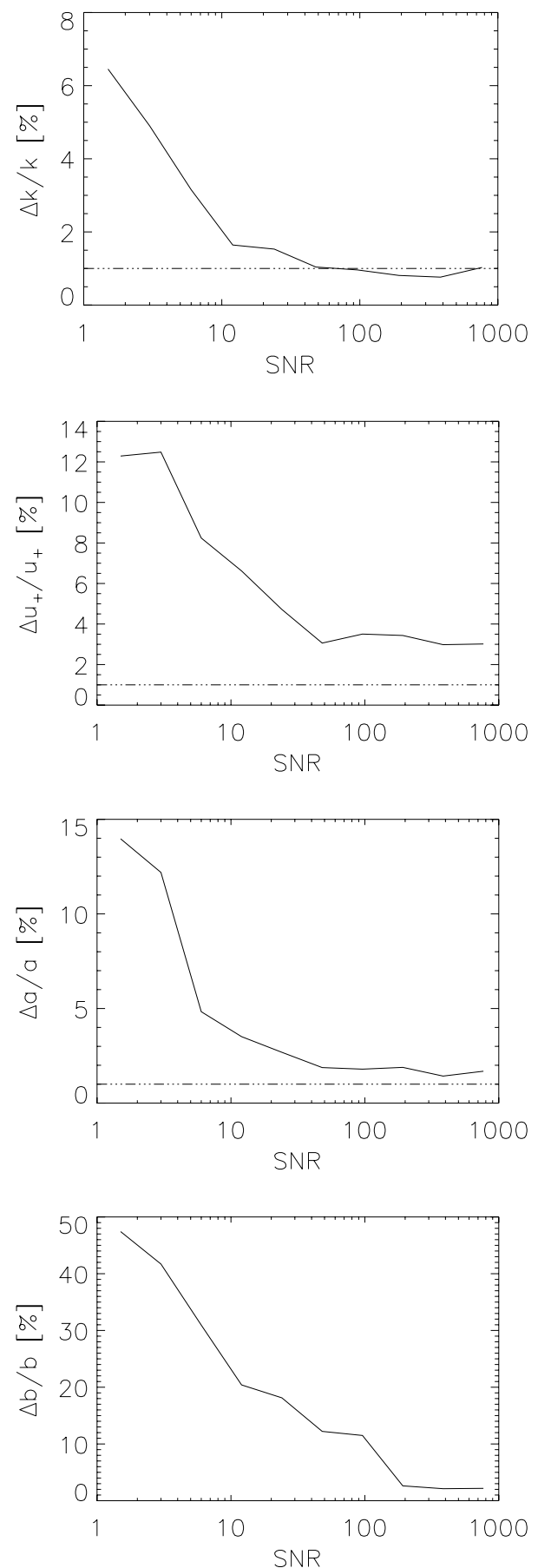

Fig. 3. Results of the light curve modelling test. On the $x$-axis the $\mathrm{S} / \mathrm{N}$ quantity is defined in the text. $y$-axes are the relative uncertainties in radius ratio $k, u_{+}$combination of the two quadratic limb darkening coefficients used, in the scaled semi-major axis (for brevity we used the notation of $a$ only instead of $a / R_{\mathrm{s}}$ in the third panel from top) and in the impact parameter $b$, from top to bottom, expressed in percentages. The solid lines show the relative uncertainties defined as the standard deviations of the differences of the modelled and the input values normalized to the input values at certain $\mathrm{S} / \mathrm{N}$-values.

do not change around and during a transit. The constancy of the aforementioned quantities are reasonable.

These distortions mean that the local effective surface temperature where they occur is lower for stellar spots and higher for faculae than that of the undistorted stellar surface. As is well 
known, the limb darkening coefficient is different for different surface temperatures. This means that the apparent stellar disc cannot be characterized by only one effective surface temperature value, and that is why the surface brightness distribution cannot be characterized by only using the limb darkening coefficients due to only one $T_{\text {eff. }}$.

Djurasevic (1992) already took into account that the undistorted and the distorted areas have different limb darkening coefficients, when he developed a code to analyse eclipsing binary star light curves. The corresponding limb darkening coefficients were taken from theoretical approximations available at that time. We would need a similar approach for the transit modelling. However, we suggest avoiding exactly the same approach for the following reasons.

a) Different tables give different limb darkening coefficients, which is why the results can be different, and maybe the derived spot parameters will depend on the assumed limb darkening coefficients.

b) The spot distribution is not well known, and the spot solution is often highly degenerated so the reliability of the spot solution can be criticized if only one-colour photometry is available. Consequently it is hard to include the spots in a modelling tool.

\subsection{Effect of a single active area}

To investigate the effect of stellar spots and faculae (hereafter we call both spots, including spots that can have higher temperatures than the surface, i.e. "bright spots", describing the characteristics of faculae that they have a higher temperature than the normal photospheric area), we classify the spots as follows. Spots that are not eclipsed by the planet we define as Type I spots and spots that are eclipsed by the planet as Type II spots.

The effect of Type II spots can be taken into account because the size and location of the spots can be determined with high accuracy, and that is why removing of their contribution from the light curve causes no problem, at least in principle (Silva-Valio \& Lanza 2011; Sanchis-Ojeda \& Winn 2011; Désert et al. 2011).

Type I spots are more complicated. Those that cause light curve modulations can be removed safely with baseline fits and baseline corrections using the out-of-transit points in the small vicinity of transits. But there are also spots that cause no light curve modulations, e.g. a polar spot. In addition, Jackson \& Jeffries (2012) propose, based on spectral evidence, that a considerable amount of stars exhibit numerous (up to 5000), small (not bigger than 2-3 astrographic degrees) dark spots that are axysymmetrically distributed on the stellar surface, reaching $\sim 50 \%$ spot-coverage, which led to no observable light curve modulation over the current detection limit in spite of their high activity level. If this is true then we can expect that even photometrically quiet stars can produce strange limb darkening coeffients as we will show.

Figure 4 illustrates the situation. When the planet moves in front of an unmaculated star, the observable maximum flux change will be

$$
\frac{\Delta F}{F_{0}}=k^{2} \frac{1-u_{10}\left(1-\mu_{\mathrm{p}}\right)-u_{20}\left(1-\mu_{\mathrm{p}}\right)^{2}}{1-\frac{u_{10}}{3}-\frac{u_{20}}{6}}
$$

where $\Delta F$ is the flux decrease due to the planet transit, $F_{0}$ is the total emitted flux of the star in our direction, $u_{10}$ and $u_{20}$ are the
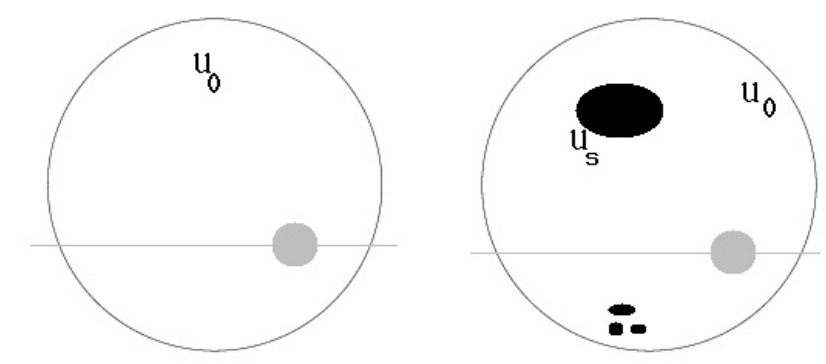

Fig. 4. Illustration of the effect of Type I spots. Left: the planet crosses an unmaculated star that is characterized with some limb darkening coefficient $u_{0}$. Right: the planet crosses the appareant stellar disc of a spotted star, where the spots and the planet have different impact parameters, as well as the stellar photosphere and the spots have different limb darkening coefficients $\left(u_{0}, u_{\mathrm{s}}\right)$. Grey area is the planet, black ellipses represent the spots.

limb darkening coefficients of the unmaculated stellar surface and index $\mathrm{p}$ stands for the planet.

If we have one circular Type I spot on the stellar surface, then this can be modelled by replacing the stellar flux with the spot's flux at the place of the spot. Then, we use the new $F_{\text {star }}$ value instead of $F_{0}$ in Eq. (3). In this case, the total flux of a spherical star with only one Type I spot can be written as (if the spot is small enough)

$$
\begin{aligned}
F_{\mathrm{star}}= & \pi R_{\mathrm{star}}^{2} I_{0}\left(1-u_{10} / 3-u_{20} / 6\right) \\
& -\pi R_{\mathrm{s}}^{2}\left[I_{0} L_{\mathrm{D}}\left(u_{10}, u_{20}, \mu_{\mathrm{s}}\right)-I_{\mathrm{s}} L_{\mathrm{D}}\left(u_{1 s}, u_{2 s}, \mu_{\mathrm{s}}\right)\right] .
\end{aligned}
$$

Index s stands for the spotted area. The first term on the right hand side describes the total flux stemming from the stellar disc, while the second term describes the effect of the spot: we remove a certain small part of the stellar flux and replace it by the spot's flux. The argument of the $L_{\mathrm{D}}$ function is $\mu_{\mathrm{s}}$ which we can calculate easily from the spot's position. This equation also shows that in the case of a dark spot, $F_{\text {star }}$ is smaller and the transit depth becomes larger. For faculae, which are brighter than the surface, $F_{\text {star }}$ become larger and hence the transit depth will be smaller.

When we model the transit, we replace this real star with a hypothetical star of an unmaculated surface. Although the transit depth will change due to the presence of stellar spots, it is easy to show that the radius ratio $k$ remains unchanged and precisely determinable if one chooses the effective limb darkening coefficients appropriately.

We use the following abbreviation (cf. Eq. (4)):

$$
A=\frac{\sum_{j=1}^{N_{\mathrm{s}}} \pi R_{\mathrm{s} j}^{2}\left[I_{0} L_{\mathrm{D}}\left(u_{10}, u_{20}, \mu_{\mathrm{s} j}\right)-I_{\mathrm{s} j} L_{\mathrm{D}}\left(u_{1 \mathrm{~s} j}, u_{2 \mathrm{~s} j}, \mu_{\mathrm{s} j}\right)\right]}{\pi R_{\mathrm{star}}^{2} I_{0}}
$$

where $j$ stands for the $j$ th spot. Then we have a step-by-step calculation of the observable light loss during the transit,

$$
\begin{aligned}
\frac{\Delta F}{F_{\text {star }}} & =k^{2} \frac{1-u_{10} \delta-u_{20} \delta^{2}}{1-\frac{u_{10}}{3}-\frac{u_{20}}{6}-A} \\
& =k^{2} \frac{1-\frac{u_{10}}{1-A} \delta-\frac{u_{20}}{1-A} \delta^{2}}{1-\frac{u_{10}}{3(1-A)}-\frac{u_{20}}{6(1-A)}}+\alpha,
\end{aligned}
$$

where we abbreviate

$$
\alpha=\frac{A k^{2}}{(1-A)\left(1-\frac{u_{10}}{3(1-A)}-\frac{u_{20}}{6(1-A)}\right)} .
$$

This $\alpha$ is constant during the transit, because it does not depend on the planet-star distance and we assumed above that the spot is 
fixed in space, i.e. the star rotational period is much longer than the transit length.

Finally the basic equation which relates the observed light loss during a transit, the planet-to-stellar radius ratio, stellar spot effects and limb darkening to each other is

$$
\frac{\Delta F-\alpha F_{\text {star }}}{F_{\text {star }}}=k^{2} \frac{1-u_{1 \mathrm{eff}} \delta-u_{2 \mathrm{eff}} \delta^{2}}{1-\frac{u_{1 \mathrm{eff}}}{3}-\frac{u_{2 \mathrm{eff}}}{6}} .
$$

Comparing the equations of this section to each other we can see that the newly defined effective limb darkening coefficients are related to the values valid on the unspotted stellar surface such that:

$u_{1 \mathrm{eff}}=\frac{u_{10}}{1-A}, \quad u_{2 \mathrm{eff}}=\frac{u_{20}}{1-A}$.

From this mathematical analysis of transit light curves it is obvious now that spots acts like a contamination source in the aperture and this is valid during the entire transit. If an unresolved star or other light source (also called "contamination source") contributes to the observed flux, then that decreases the observed transit depth. This extra light source is routinely removed from the light curve in general. Certain spots acts as a negative $\alpha$. In total, this kind of contamination-like source can be either positive or negative, but in the transit fits we did not include negative contamination.

It has been already recognized, that Type I spots can act as contamination sources, but our result implies also that limb darkening coefficients can also change which is a new result. In addition to the arbitrary sign of $\alpha$, these latter two equations justify that contamination should be an additional free parameter in the transit fit which would enable to remove all the spot-effects which may remain after the baseline-correction since they might not cause light curve modulation in and out of transit. It also shows that no detailed spot modelling is needed to fully understand the transit light curves if no spot-crossing occurs, even if polar or any other spots are present. The problem of this case can be solved in the following simple and robust way: limb darkening coefficients and contamination factor should be free parameters. Last but not least it shows that the observed limb darkening coefficients can be highly different from the theoretically predicted values in the presence of spots, even if they do not produce light curve modulations. The uniqueness of the transit light curve solutions are provided by the fact that the length of the ingress and egress phases, as well as the full transit length, are also related to the size ratio of the star and the planet (see e.g. Winn 2010).

This analysis is only valid for small spots. The analysis can, however, be generalized easily for numerous and larger spots, as we did with the summation above. It is also easy to extend the analysis to bright spots (i.e. faculae). If one takes more terms in the expression of the limb darkening laws into account, then the effective limb darkening coefficients will be transformed in the same way as the first two terms, and it is then clear how one has to extend the $\alpha$-function with additional limb darkening terms.

We also note that for this spotted case the same applies as for the previous unspotted case in Sect. 4: if the limb darkening coefficients are fitted, then we can determine their exact values if they fulfil the $\mathrm{S} / \mathrm{N}$ requirements. We have to keep in mind, however, that we measure the effective limb darkening coefficients in this case and not the ones related to an unmaculated stellar surface.

Recently, combined optical and near infrared observations of transits were proposed by Ballerini et al. (2012) to minimize the effect of spots on the transit parameters. It was proven therein that it is possible to derive precise planetary radii from this kind of multicolour observation, if the temperature of spots and the theoretical limb darkening coefficients are known. Here we have showed that the same result can be reached without spot parameters and without the knowledge of the theoretical limb darkening coefficients (which are practically unkown today). Our method is not only simpler, but it does not require multicolour photometry. Both methods have the advantage of being able to provide the exact and precise planetary parameters in highly active cases when the spots are not crossed by the planet, and therefore we have limited information about the stellar surface.

\subsection{Effect of more than one active area}

The direct observations of the Sun's surface, the Dopplerimaging of other active stars and their photometric spot studies show concordantly that, in general, more than one active area is present on the stellar surface at the same time. The results of the previous section can be generalized easily by simple summing of the effects of several individual active areas (cf. Eq. (5)).

Studies of the Sun show that the total area of the faculae depends on the area of the dark spots. According to Chapman et al. (1997), the following expression is a good approximation for the Sun

$\Sigma A_{\text {faculae }} \approx 17 \Sigma A_{\text {spot }}$,

but the conversion factor of the total area ratio shows small secular variations during one solar cyle (Chapman et al. 1997). It is reasonable to assume that other stars have similar relationships between their total area ratios, although the conversion factor in Eq. (10) can vary. For instance, Chapman et al. (2011) find that the conversion factor varies from cycle to cycle, and for solar Cycle 23 it was 42 instead of the 17 that is valid for Cycle 22. More studies are required to determine this factor and its dependence on the stellar properties. Foukal (1998) shows that there is an anticorrelation between $A_{\text {spot }}$ and $A_{\text {faculae }}$ in the case of the Sun and he argues that this can be extrapolated to other stars.

The total spot coverage varies over a wide range. The Sun has a spot coverage of $0.1 \%$ only at maximum. Several observations suggest that many of the normal stars can have $1-2 \%$ spot coverage, but there are quite a few stars showing 10-60\% spot coverage on its surface (Schrijver \& Zwaan 2004, and references therein; this coverage refers to the total area of the spots relative to the whole stellar surface). The most active stars seem to be located in close binary stars and giants, and they are not so important from the point of view of the presently known transiting exoplanets. But some exoplanet hosting stars (like CoRoT-2, CoRoT-6, CoRoT-7 etc.) seem to be very active stars. E.g. Lanza et al. (2009) established 7\% spot coverage for CoRoT-2.

The changes in the limb darkening coefficients have dramatic conclusions for the errors in planet-to-stellar radius ratio if one fixes the limb darkening coefficients according to tabulated values and neglects the effect of spots on the limb darkening. If one accepts the conclusions of Jeffries \& Jackson (2012), namely that $\sim 50 \%$ of the surface can be covered by small spots in an active star without observable photometric modulation (but spectroscopic activity indicators still imply an active nature of this hypothetical star), then the $A$-function defined by Eq. (5) can have a value of $\sim 0.36$. This causes via Eq. (9) the observable limb darkening coefficients to increase by a factor of 1.56 - if we do not take into account the role of faculae, which may be negligible according to the extrapolation suggested by 
Foukal (1998). If the host star is less active, we take 5\% spot coverage as an example, the $A$-function will cause a change of $\sim 0.037$ in the limb darkening coefficients, which will lead to $9 \%$ error in the radius ratio (cf. Eq. (1)). If the star has only $0.5 \%$ spot coverage, then the $A$-function will cause a change of only 0.004 in the limb darkening coefficients, but this still causes $1 \%$ error in the radius ratio. Thus, $0.5 \%$ spot coverage can be considered as a limit: below it one can neglect the change in the limb darkening coefficients owing to Type I stellar spots. These changes in the observed effective and the tabulated limb darkening coefficients are plotted in Fig. 5. (For this estimation we used a rather grazing eclipse. For central eclipses the effect is less but still considerable.)

We note that when a host star of a transiting planet is highly active, like CoRoT-2, then it is not enough to take an appropriate contamination factor into account to change the transit depth or to carefully model the crossing of Type II spots, including a baseline correction to remove all the contributions of spots to the photometry. It is also necessary to keep the limb darkening coefficients as free parameters or to modify them according to Eq. (9), because the modification of the limb darkening coefficients will also cause non-negligible effect on the planetary radius.

Thus we conclude that stellar activity modifies both the observable effective limb darkening coefficients, as well as the observable transit depth. Then, if spots and faculae are present, one cannot fix the limb darkening coefficients corresponding to the stellar effective surface temperature. It is preferable to leave them as adjustable parameters, because it is difficult to predict the effect of the spots on the limb darkening coefficients. We also established that the effective limb darkening coefficients (note that almost all transit light curve modellers use these) can have unphysical values because of spots and faculae. Of course, "unphysical" is only the correct word if, and only if, we do not compare the spotted star to an unspotted model calculation. It is also clear that in such a case, we also can determine with good accuracy $(<1 \%)$ the radius ratio of the star and the planet, the impact parameter, and the $a / R_{\mathrm{S}}$ ratio and the limb darkening coefficients, but we just keep in mind, that in the case of several spots and faculae, the limb darkening coefficients yielded by the modelling do not correspond to the limb darkening coefficients of an unmaculated star with the same effective temperature, but instead correspond to the effective limb darkening coefficients.

\section{Determinability of individual limb darkening coefficients}

Here we study the determinability of the specific limb darkening coefficients. Since many combinations of limb darkening coefficients are able to reproduce the same surface brightness distribution within the errors of the observations, it seems likely that the light curve fitting procedures reproduce the intensity distribution and not the true values of the coefficients. In other words, the inversions of the polynomial expressions of the limb darkening laws incorporate many degeneracies. That is why we are able to determine all the transit parameters with good accuracy and to reproduce the corresponding stellar surface brightness distribution within the errors of the observations, but we are not able to determine the individual values of the limb darkening coefficients. From the point of view of exoplanet parameter determination this does not present any difficulties. To illustrate this, we

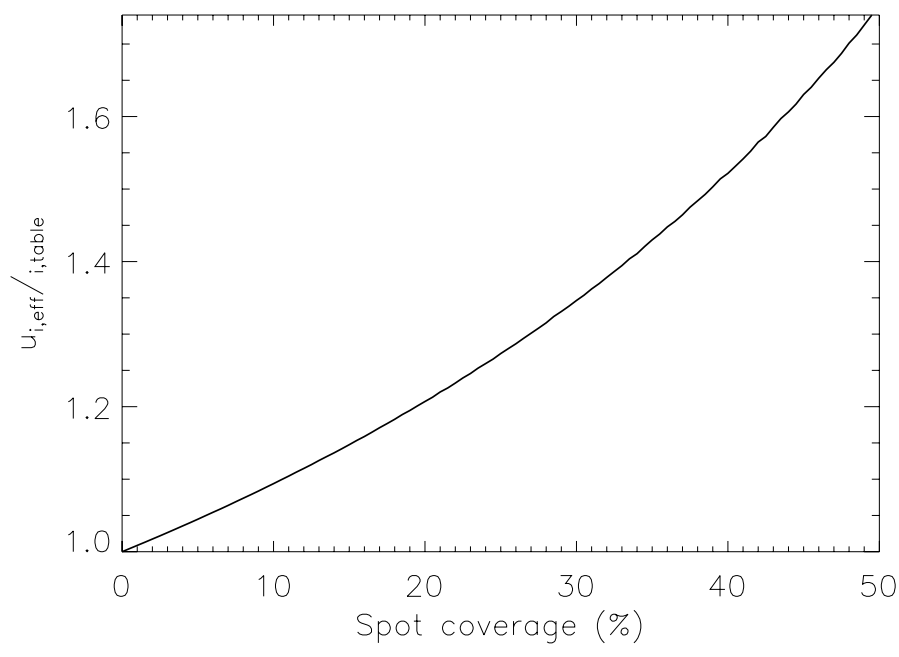

Fig. 5. The $x$-axis is the total spotted area in percentage of the whole stellar surface area. The $y$-axis is the effective - i.e. observed - limb darkening coefficients relative to the table value at the given stellar efffective temperature. For this figure we used $T_{\text {star }}=5775 \mathrm{~K}$ and $T_{\text {spots }}=3775 \mathrm{~K}$, and the positions of the spots were chosen randomly on the visible hemisphere. The size of the spots were always the same, so higher spot coverage corresponds to larger number of spots. The limb darkening coefficients were taken from Claret \& Bloemen (2011) for $R$-band, $\log g=4.5$ and at solar metallicity at these temperatures. Effects of faculae were not considered in this figure.

rewrite Eq. (1) for the following analysis as

$k^{2}=\frac{\Delta F / F}{L_{\mathrm{D}}}$.

The current aim is to know the planetary radii with $5 \%$ precision (Valencia et al. 2007), although the future goal is $1 \%$ (Wagner et al. 2011). To determine $k$, say, with $5 \%$ precision, it is enough to know the limb darkening with a precision of $10 \%$, which follows from the linear error analysis

$\frac{\Delta k}{k}=\frac{1}{2}\left(\frac{\Delta F}{F}+\frac{\Delta L_{\mathrm{D}}}{L_{\mathrm{D}}}\right)$

because the photometric error is practically negligible for spacebased photometry beside the error steming from limb darkening's errors (cf. Sect. 3). This means that the transit light curve modelling codes can produce quite different limb darkening coefficients than what we expect. Now we aim to determine these differences.

We denote the expected limb darkening coefficients by $u_{i}$, and the difference between the measured and the expected values by $\beta_{i}$. The relative deviation between measured and expected stellar intensity profiles is denoted by $\epsilon(\delta)$ and the maximum allowed relative deviation - occurs at the still unknown $\delta$ - is denoted by $\epsilon_{\max }$, so we can write

$L_{\mathrm{D}}\left(u_{1}, u_{2}, \ldots, \delta\right)-L_{\mathrm{D}}\left(u_{1}+\beta_{1}, u_{2}+\beta_{2}, \ldots, \delta\right)=\epsilon_{\max }$.

Although the analysis can easily be extended to higher orders, we cut it at second order because this is generally used for transit analysis (but as can be seen, the conclusion does not change significantly if one extends the calculation to higher orders).

We chose a solar-like star to illustrate the operation of the $\beta_{i}$ numbers. We plotted the deviation $|\epsilon|$ for different cases in Fig. 6. In that figure, there is a rather wide range for $\beta_{i}$ parameters that produce the same limb darkening function within $\pm \epsilon$ tolerance 


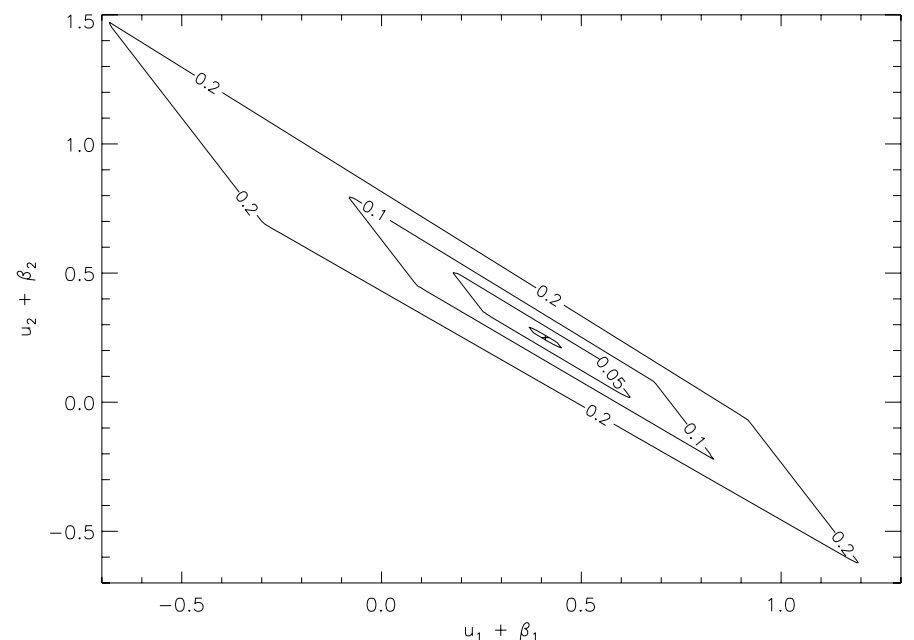

Fig. 6. The $x$-axis is the modified linear limb darkening coefficient $x_{1}=u_{1}+\beta_{1}$, the $y$-axis is the modified quadratic limb darkening coefficients $x_{2}=u_{2}+\beta_{2} . u_{1}, u_{2}$ are fixed. The point in the middle shows the $u_{1}=0.41, u_{2}=0.25$ point, i.e. the original value of the limb darkening coefficients of the example presented in Sect. 8. Contours represent the quantity $\epsilon_{\max }$, i.e. the absolute value of the maximum deviation of the original limb darkening function with $u_{1,2}$ coefficients from the limb darkening function with $x_{1,2}(\delta$ run between 0 and 1$)$.

range. It is easy to construct an $L_{\mathrm{D}}$ function, for example, of limb darkening coefficients of $u_{1}+\beta_{1}=0.8$ and $u_{2}+\beta_{2}=-0.1$ and this produces the same results within the tolerance range as the correct values $u_{1}=0.41$ and $u_{2}=0.26$. This example is presented in Fig. 6. Along the sides of the paralellograms one can easily construct the same quality of the light curve fit (cf. Eq. (13)). In Fig. 7 we plotted another example: the stellar surface brightness distribution for different $\beta_{i}$ numbers. As one can see there is a high tolerance range, so one can choose very different limb darkening combinations from the theoretical predictions that produce a similar surface brightness distribution within the tolerance range. This example shows that we are able to produce acceptable $D(r / R=\mu)$ functions within the tolerance ranges with quite different limb darkening coefficients. If the tolerance range changes due to different precision requirements, the acceptable range of the limb darkening coefficients will also change.

One can envisage that a transit light curve modelling code may take different numbers as input for numerical reasons or owing to data points/error distributions than the theoretically expected values. This can be also the case if stellar spots modify the limb darkening coefficients. That is why the absolute values of the limb darkening coefficients are not important but the shape of the radial intensity distribution that they define is (see Fig. 7 for such a comparison).

This analysis gives hints and arguments that what we derive from the transit light curve are not the true individual values of the limb darkening coefficients but some rather complicated combination of them. The limits of the potentially wide ranges depend on the actual impact parameter and photometric precision (i.e. the range of the tolerance factor $\epsilon$ ), so that to give the limits requires case studies.

From Fig. 6 it is also clear that the fitted limb darkening coefficients are also correlated with each other. This behaviour has already been observed by numerical experiences in Brown et al. (2001) and in e.g. Deleuil et al. (2008) and Csizmadia et al. (2011). Brown et al. (2001) and Pál (2008) suggest fitting the combination $u_{+}=u_{1}+u_{2}, u_{-}=u_{1}-u_{2}$ and

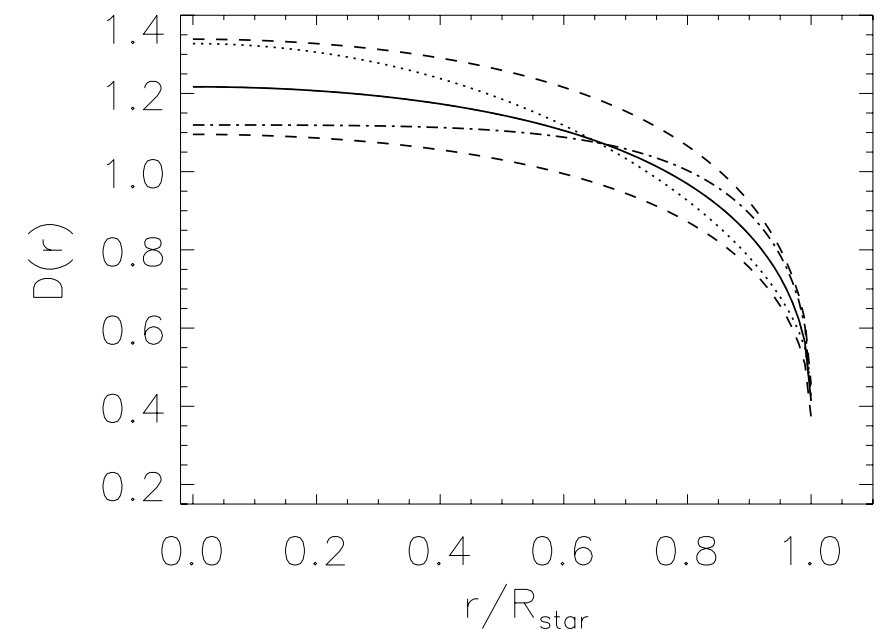

Fig. 7. The radial intensity profile $D(r / R)$ as a function of the skyprojected distance $r$ from the stellar centre ( $R$ is the stellar radius). As in all calculation in this paper, $\mu=\sqrt{1-(r / R)^{2}}$. The solid line shows the effect of limb darkening $D(r)=L_{\mathrm{D}}\left(u_{1}, u_{2}, \mu\right) /\left(1-u_{1} / 3-u_{2} / 6\right)$ that multiplied by $k^{2}$ - is directly proportional to the light loss during a transit. The dashed lines show the tolerable ranges: between these lines the radial intensity distribution profile will produce a radius ratio $k$ that is in the tolerance range of $\pm 5 \%$. The dotted line is an example of an acceptable radial intensity distribution profile with $u_{1}=0.82, u_{2}=-0.16$, while the dot-dashed line is with $u_{1}=0.02, u_{2}=0.6$.

$u_{+}=u_{1} \cos 40^{\circ}+u_{2} \sin 40^{\circ}, u_{-}=u_{1} \cos 40^{\circ}-u_{2} \sin 40^{\circ}$ instead of fit $u_{1}$ and $u_{2}$ themselves because they are less correlated to each other as previously assumed. As is clear from Fig. 6 this rotational-like transformation can help better constrain one of these combinations, but the other will still feature a value in a wide range and the problem cannot be surpassed in this way. The values that look "unphysical" at first sight may describe the radial intensity distribution as well as do other values. We emphasize again that the absolute values of the fitted limb darkening coefficients are themselves not important but the radial intensity profiles are. These profiles, taking the tolerable deviations into account, can be produced with a very wide ranges of limb darkening coefficients. That is why the basis of the analysis of the results are not comparison of the resulting limb darkening coefficient values themselves to some theoretical table values, but the comparison of the resulting radial intensity profiles of the predicted with the observed ones, a diagram similar to Fig. 7.

\section{Conclusions}

In this paper we have attempted to understand the impact of limb darkening on the planetary radius, to present ideas as to which effects can modify the theoretically predicted limb darkening coefficients, and to answer why some authors have highly different observed limb darkening coefficients from the theoretical predictions.

We concluded that concurrent limb darkening tables do not yield consistent results in several temperature- and metallicity regions, and we showed that the inconsistencies will lead to serious defects in the determination of the planet-to-stellar radius ratios if one fixes the limb darkening coefficients according to these tables. We also proved with numerical experiments that one can safely fit the limb darkening coefficients.

We investigated how the presence of stellar spots - both dark spots and bright faculae - modify the observable transit 
depth and the limb darkening coefficients. We found that below $0.5 \%$ spot coverage, the change in the effective limb darkening coefficients causes no significant error in the measured planet-to-stellar radius ratio, but over that coverage this cannot be neglected, and neglecting the change in the limb darkening coefficients themselves has a significant change in the radius ratio.

We pointed out that the radial intensity profiles of the stellar discs are not very sensitive to the specific values of the limb darkening coefficients, and they are anticorrelated to each other in the case of the quadratic limb darkening law (cf. Fig. 6), and a similar statement can be made about higher order limb darkening laws. Consequently, the observed values of the limb darkening coefficients can be very different from the theoretically tabulated ones even if they produce the same radial intensity profiles within the tolerance.

Limb darkening is still a problem for transit modelling. On the one hand, limb darkening is a challange for stellar astrophysics. On the other hand, we can replace the limb darkening coefficients of an unmaculated star with the appropriately weighted effective limb darkening coefficients for the modelling. Our final conclusion is that the transit parameters can be determined even if our knowledge of limb darkening and stellar spots is not satisfactory. Our work will make it clear that one has to fit the limb darkening coefficients and it is not self-evident that the resulting limb darkening coefficients should agree with the theoretical predictions. Indeed, these can be very different. Our results suggest not only that fitting the limb darkening coefficients will produce more reliable solutions but also that their adjustment will not affect the accuracy of determining other transit parameters. However, predicting the effective limb darkening coefficients is difficult, because it requires a reliable and detailed spot solution. Moreover, these can vary from transit to transit as the spots evolve. If we fit them, then we can determine their values, too, but then such effective limb darkening coefficients have no direct link to the theoretical limb darkening tables. When a statistically significant sample of high-quality, multicolour photometric analyses of transits become available, then the comparison of the resulting limb darkening coefficient to the available tables will become useful to characterize the discrepancies between theory and observations better. This can contribute significantly to improving our knowledge of stellar atmospheres. It is also important to simultaneously determine the activity level of the star and the limb darkening coefficients

It is also clear that further detailed studies are required to completely understand the limb darkening phenomenon and its interaction with e.g. gravity darkening. Such studies are very useful for planning such projects as PLATO or for understanding the present-day ground- and space-based measurements more deeply.

Acknowledgements. This research has made use of the SIMBAD database, operated at the CDS, Strassbourg, France. We thank an anonymous referee for his/her comments.

\section{Appendix A: The limb darkening laws}

Limb darkening is a stellar atmosphere phenomenon that is reviewed in detail in several textbooks (see e.g. Kallrath \& Milone 2009). The most general formula to describe the effect of limb darkening is

$$
\begin{aligned}
I(\gamma) & =I_{0} L_{\mathrm{D}}\left(u_{1}, u_{2}, \ldots, u_{i}, \ldots, \mu\right) \\
& =I_{0}\left(1-\Sigma_{j=1}^{\infty} u_{j}(1-\cos \gamma)^{j}\right),
\end{aligned}
$$

where $u_{j}$ are the so-called limb darkening coefficients, $\gamma$ the angle between the normal vector of the surface point and the direction to the observer, $I_{0}$ and $I(\gamma)$ are the surface brightnesses (or intensities) at the appearant centre of the stellar disc and in a certain point of the stellar surface characterized by the angle $\gamma$, and $L_{\mathrm{D}}$ is the limb darkening function defined by Eq. (A.1). We also use the common notation $\mu=\cos \gamma$.

Many studies of eclipsing binaries have indicated that at least two terms in Eq. (A.1) should be kept to fit the light curves satisfactorily (Twigg \& Raffert 1980; Alencar \& Vaz 1999; Albrow et al. 2001; Claret 2000, 2008, Southworth et al. 2007). That is why, in the exoplanetary research field it is widely accepted to use the truncated formula of Eq. (A.1)

$L_{\mathrm{D}}=1-\Sigma_{j=1}^{2} u_{j}(1-\cos \gamma)^{j}$,

which can easily be rewritten as

$L_{\mathrm{D}}=1-\left(u_{1}+u_{2}\right)+\left(u_{1}+2 u_{2}\right) \mu-u_{2} \mu^{2}$.

There are different approaches describing the limb darkening effects, including logarithmic or square root terms of $\mu$, see e.g. Claret (2000) or van Hamme (1993) for an overview.

\section{Appendix B: Transit depth and limb darkening}

It is straightforward to show in the small-planet approximation that the transit depth can be written as

$$
\frac{\Delta F}{F}=k^{2} \frac{1-u_{1}(1-\mu)-u_{2}(1-\mu)^{2}}{1-\frac{u_{1}}{3}-\frac{u_{2}}{6}},
$$

where

$\Delta F=\pi R_{\text {planet }}^{2}\left(1-u_{1}(1-\mu)-u_{2}(1-\mu)^{2}\right)$

and

$F=\pi R_{\text {star }}^{2}\left(1-\frac{u_{1}}{3}-\frac{u_{2}}{6}\right)$

also hold, and it is trivial to extend these expressions to higher orders of the limb darkening approximations (cf. Kallrath \& Milone 2009; Mandel \& Agol 2002). In these expressions $\Delta F$ is the light lost due to the transit; $F$ is the stellar flux out of the transit part of the light curve; $k=R_{\text {planet }} / R_{\text {star }}$ is the ratio of the planet radius $R_{\text {planet }}$ and the star's radius $R_{\text {star }}$. In addition, in the moment of the maximum light lost, $\mu=\sqrt{1-b^{2}}$ where $b$ is the impact parameter. If we are interested in the light loss at any arbitrary time inside the transit, then $\mu=\sqrt{1-\delta^{2}}$ where $\delta$ is the sky-projected distance measured in stellar units. (For details on how to determine it for any arbitrary time see Kallrath \& Milone 2009.)

Taking the typical values of the limb darkening coefficients into account (see Sect. 2 or the references mentioned in Sect. 1), one can easily see that limb darkening will modify the true depth by up to a factor of $\sim 2$ relative to the case where one takes the transit depth to be proportional only to the radius ratio - therefore the effect of limb darkening is not negligible.

\section{References}

Albrow, M. D., An, J., Beaulieu, J. P., Caldwell, J. A. R., \& Depoy, D. L. 2001, ApJ, 549, 759

Alencar, S. H. P., \& Vaz, L. P. R. 1999, A\&AS, 135, 555

Ballerini, P., Micela, G., Lanza, A. F., \& Pagano, I. 2012, A\&A, 539, A140

Barban, C., Goupil, M. J., Van't Veer-Menneret, C., et al. 2003, A\&A, 405, 1095 
Sz. Csizmadia et al.: Transit parameters, stellar spots and limb darkening

Barnes, J. W. 2009, ApJ, 705, 683

Barros, S. C. C., Pollacco, D. L., Gibson, N. P., et al. 2012, MNRAS, 419, 1248

Bordé, P., Bouchy, F., Deleuil, M., et al. 2010, A\&A, 520, A66

Brown, T. M., Charbonneau, D., Gilliland, R. L., Noyes, R. W., \& Burrows, A. 2001, ApJ, 552, 699

Bruntt, H., Deleuil, M., Fridlund, M., et al. 2010, A\&A, 519, A51

Cabrera, J., Bruntt, H., Ollivier, M., et al. 2010, A\&A, 522, A110

Catala, C. 2009, Comm. Asteros., 158, 330

Chapman, G. A., Cookson, A. M., \& Dobias, J. J. 1997, ApJ, 482, 541

Chapman, G. A., Dobias, J. J., \& Arias, T. 2011, ApJ, 728, 150

Clausen, J. V., Bruntt, H., Claret, A., et al. 2009, A\&A, 502, 253

Claret, A. 2000, A\&A, 363, 1081

Claret, A. 2004, A\&A, 428, 1001

Claret, A. 2008, A\&A, 482, 259

Claret, A. 2009, A\&A, 506, 1335

Claret, A. 2012, A\&A, 538, A3

Claret, A., \& Bloemen, S. 2011, A\&A, 529, A75

Claret, A., \& Hauschildt, P. H. 2003, A\&A, 412, 24

Claret, A., Diaz-Cordoves, J., \& Gimenez, A. 1995, A\&AS 114, 247

Csizmadia, Sz., Moutou, C., Deleuil, M., et al. 2011, A\&A, 531, A41

Deleuil, M., Deeg, H. J., Alonso, R., et al. 2008, A\&A, 491, 889

Deleuil, M., Bonomo, A. S., Ferraz-Mello, S., et al. 2012, A\&A, 538, A145

Désert, J. M., Charbonneau, D., Demory, B. O., et al. 2011, ApJS, 197, 14

Diaz-Cordoves, J., Claret, A., \& Gimenez, A. 1995, A\&AS, 110, 329

Djurasevic, G. 1992, Ap\&SS, 196, 241

Espinoza, L. F., \& Rieutord, M. 2011, A\&A, 533, 43

Foukal, P. 1998, ApJ, 500, 958

Gandolfi, D., Hébrard, G., Alonso, R., et al. 2010, A\&A, 524, A55
Geem, Z. W., Kim, J. H., \& Lonatathan, G. V. 2001, Simulation, 76, 60 Gillon, M., Hatzes, A., Csizmadia, Sz., et al. 2010, A\&A, 520, A97

Hayek, W., Sing, D., Pont, F., \& Asplund, M. 2012, A\&A, 539, A102 Howarth, I. D. 2011, MNRAS, 413, 1515

Jackson, R. J., \& Jeffries, R. D. 2012, MNRAS, 423, 2966

Kallrath, J., \& Milone, E. F. 2009, Eclipsing Binary Stars: Modeling and Analysis (Springer)

Kipping, D., Bakos G. 2011, ApJ, 733, 36

Lanza, A. F., Pagano, I., Leto, G., et al. 2009, AIPC, 1094, 684

Mandel, K., \& Agol, E. 2002, ApJ, 580, 171

Neilson, H. R., \& Lester, J. B. 2011, A\&A, 530, A65

Pál, A. 2008, MNRAS, 390, 281

Ribas, I., Morales, J. C., Jordi, C., et al. 2008, MmSAI, 79, 562

Sanchiz-Ojeda, R., \& Winn, J. N. 2011, ApJ, 743, 61

Schrijver, C. J., \& Zwaan, C. 2004, Solar and Stellar Magnetic Activity (Cambridge University Press)

Southworth, J. 2011, MNRAS, 417, 2166

Southworth, J., Bruntt, H., \& Buzasi, D. L. 2007, A\&A, 467, 1215

Silva-Valio, A., \& Lanza, A. F. 2011, A\&A, 529, A36

Sing, D. K. 2010, A\&A, 510, 21

Torres, G., Andersen, J., \& Giménez, A. 2010, A\&ARv 18, 67

Torres, G., Fischer, D. A., Sozetti, A., et al. 2012, ApJ, 757, 161

Twigg, L. B., \& Raffert, J. B. 1980, MNRAS, 193, 775

van Hamme, W. 1993, AJ, 106, 2096

von Zeipel, H. 1924, MNRAS, 84, 665

Valencia, D., Sasselov, D. D., \& O’Connell, R. J. 2007, ApJ, 665, 1413

Wagner, F. W., Sohl, F., Hussmann, H., Grott, M., \& Rauer, H. 2011, Icarus, 214, 366

Winn, J. N. 2010 [arXiv.org: 1001.2010] 